\title{
A Traffic Light Recognition Algorithm Based On Compressive Tracking
}

\author{
Xuanru Zhou, Jiazheng Yuan and Hongzhe Liu \\ Beijing Key Laboratory of Information Service Engineering, Beijing Union \\ University \\ zhouxuanru1989@163.com,jiazheng@buu.edu.cn,liuhongzhe@buu.edu.cn
}

\begin{abstract}
According to the traffic light recognition problem of intelligent vehicle, this paper proposes a traffic light recognition algorithm based on compressive tracking. First, the candidate regions of traffic light are extracted. Second, after extracting the HOG feature of candidate regions, using the machine learning for classification and recognition, compressive tracking algorithm is used to track lights that have been identified a automatically. This algorithm combines feature recognition and tracking identification of traffic light and traffic light changes in scale and color can be identified normally. The algorithm proposed in this paper has been tested on actual road in intelligent vehicle; traffic light recognition effect is good.
\end{abstract}

Keywords: Compressive sensing compressive tracking traffic light identification

\section{Introduction}

In recent years, intelligent transportation has got a great development, intelligent vehicles are attracting people's attention gradually. Traffic light recognition as part of the intelligent vehicle system platform plays an important role. Traffic lights are small on video, and have scale change, their features are not rich. The mounted camera may jitter when the vehicle is on the road, and it is susceptible to the interference of complex background. These problems have to be solved. Vehicle collision could be caused by traffic light recognition errors, Casualties may happen seriously. The using of machine vision technology to track and recognize the traffic light can reduce occurrence of the situations above effectively, and it can assist intelligent vehicle safety driving efficiently.

Domestic and international researchers have done a lot of researches about traffic light recognition. Many of those are based on the recognition of the traffic light features. The features are color, shape features and so on. Usually the color spaces used in these methods include HSV [1], RGB [2, 3], and Lab [4], HIS [5] and so on. Sometimes circle feature [610], circular outer rectangle feature [3], traffic light pole geometry [11], radial symmetry feature [12] may be used. Researchers generally combine these features together to identify traffic light. Template matching [13] may be used when identification has been completed. One kind of the method to identify the traffic light is using machine learning, such as SVM [14-15] classification which bases on geometric feature and LBP (local binary pattern) feature. The most suitable method for intelligent vehicle is the priori map [16-17], as used by Google intelligent vehicle. But it will coast lots of manpower and material resources to draw the map. In these methods above, traffic light will be identified once every frame has been inputted. This will coast plenty of time. So some researchers use tracking method to achieve rapid recognition effect. For example, a method based on particle filter [18] tracking, algorithm based on CAMSHIFT [19], etc. This particle filtering method is used in railway traffic light tracking. The background in this method is too simple to overcome the interference of complex background. Camshift achieves the goal by tracking the color of moving target, but it is easy to lose the target when the inter frame displacement is large. 
Therefore, a kind of traffic light identification algorithm based on compressive tracking has been proposed in this paper. Machine learning is used to identify, then compressive tracking method [20] is used for real-time tracking. The application environment of the compressive tracking is that the camera is static and the tracking window size is constant. The algorithm has not been used for traffic light tracking. Compressive tracking is used for traffic light tracking in real-time motion in this paper. The camera moves with the vehicle. This algorithm combines the machine learning to achieve the goal that the window can change adaptively. It overcomes the problem that the tracking window size doesn't change, and has an accuracy and real-time performance.

\section{Identification Based on Features}

There are a few difficulties in traffic light identification, for example, scale change is large, light environment is complex, camera may shake. Therefore, the algorithm combines light features with classifiers to identify, and filters out interference through its own features firstly, and then uses the classifier on the remaining region to recognize. Not only algorithm running time is saved, but also the accuracy is improved.

\subsection{Color Segmentation}

Color is the most prominent feature of the traffic light, therefore, this paper preliminary locate traffic light based on color. RGB is the most common color space, but RGB space is affected by light strongly, video is affected by the extent of exposure and aperture. This space should not be used to identify lights. HIS color space is more consistent with human visual characteristics than RGB color space, and it is widely used by algorithms in image processing and machine vision. $\mathrm{H}$ represents hue, $\mathrm{S}$ for saturation, I on behalf of the brightness, the dependency of its three components is small, so it is beneficial for color segmentation. Therefore, this paper uses HIS color space. Conversion formula is as follows:

$$
\begin{aligned}
& H=\left\{\begin{array}{l}
\theta, \mathrm{G} \geq B \quad \text { where } \theta=\cos ^{-1}\left\{\frac{(R-G)+(R-B)}{2 \pi-\theta, G<B}\right\} \\
S=1-\frac{3 \mathrm{~min}(R, G, B)}{R+G+B} \\
I=\frac{R+G+B}{3}
\end{array}\right)
\end{aligned}
$$

We first get the red and green areas in the video image after the conversion. As the yellow and red color is close to each other in the extraction process, camera exposure and other reasons, the algorithm cannot separate red and yellow completely. Moreover if the yellow areas are recognized as reds, the impact is small, so only the red and green lights can be recognized in this article. $\mathrm{H}$ range $0-25$ or $330-360$ and $\mathrm{S}>=0.2$, if the area's color is red. $\mathrm{H}$ green range 160-220 and $S>=0.1$, if the area's color is green. After color extraction, the initial traffic light region is determined.

\subsection{Morphological Operations}

General morphological operations refer to corrosion and expansion, we use the morphological closing operation, this operation can fill small hole in the objects, connect to nearby objects, smooth border, but it does not significantly change its size, so it can remove noise effectively.

\subsection{Shape Segmentation}

In addition to the color characteristics, shape features are more obvious. Traffic lights' design are standardized, shape features can be used to extract their region. After closing 
arithmetic operations, traversing all the contours in binary image, this algorithm screens out the area in line with lights according to the contour features.

The first is to calculate each contour's area; the purpose is to filter out some isolated points and the area with larger area. Assuming there are $N_{r}$ candidate image regions, denoted as $R_{i}, i=1, \mathrm{~L} N_{r}$. A represents the contour area. Area which meets the following conditions will be maintained. Due to changes in the environment, the distance between the vehicle and the traffic light is not fixed; the threshold is determined by the specific driving environment.

$$
\text { Bool }\left(R_{i}(\mathrm{~A})\right)=\left\{\begin{array}{l}
1, \mathrm{~A}_{\max } \leq A \leq \mathrm{A}_{\min } \\
0
\end{array}\right.
$$

Then we get a circumscribed rectangle of each profile, according to the length, width of circumscribed rectangle, calculate aspect ratio. If the aspect ratio is too large, contour may be determined as not a traffic light.

$$
\text { Bool }\left(R_{i}\left(R_{w h}\right)\right)=\left\{\begin{array}{l}
1,0.8 \leq R_{w h} \leq 1.5 \\
0
\end{array}\right.
$$

Finally, the regions satisfied all the above conditions are reserved, called identification areas.

\subsection{Feature Extraction}

Histogram of Oriented Gradient, abbreviated as HOG, is a very common way of describing local texture features in computer vision and pattern recognition. After above steps, the remaining identification areas are relatively small. Because HOG is a local feature, only when the image area is small, histogram based on the principle of statistics for the region can express it well, so this paper uses HOG feature. At the same time, HOG is the statistic of gradient information essentially, and the gradient mainly exists in the edge. Because the edge of the traffic light board is obvious, the HOG feature is applied in feature extraction.

The region obtained above is only the light region with color of traffic lights. The characteristic of the region is not sufficient to describe the traffic light. So it is necessary to calculate the rectangular region including the board of the traffic light and extract the feature of the region to identify. The center coordinates of the region to be recognized can be obtained, the height and width of the circumscribed rectangle are already known, it is able to calculate the rectangular board region of the red and green traffic light according the shape feature.

\subsection{Classifier Training and Recognition}

SVM is a widely used statistical learning method in the field of pattern recognition and is a common method of machine learning when the training samples are limited. A lot of road video has been collected using the vehicle mounted camera, and the picture of traffic light has been tailored as training samples, the HOG feature has been extracted and 2 kinds of classifiers have been trained (red light and green light).

When the feature of the regions to be recognized has been extracted, the corresponding classifier based on the color of the region will be chosen to recognize. The result of the recognition includes color, shape and location information of the traffic light. Rectangle frame has been used to mark out the identified traffic light in this paper, as shown in Figure 5 and Figure 6. 


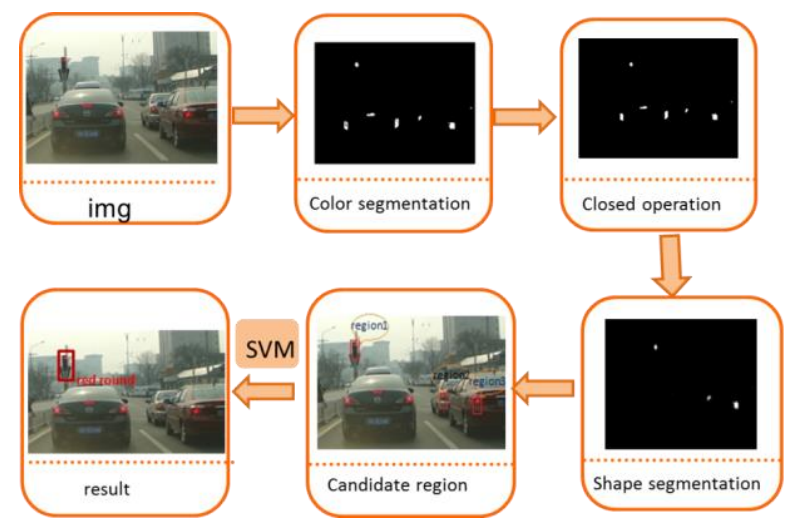

Figure 1. The Schematic of the Identification Process

\section{Recognition Based On Compression Tracking}

The background is complex because the traffic light tracking is conducted in the real town roads, and real-time is required. Tracking grift even missing phenomenon will appear when using common tracking algorithms. Compression tracking algorithm has been used in this paper to solve the mentioned problems effectively. Random sensing matrix consistent with compressive sensing RIP conditions will be used to reduce the dimension of the multiscale image feature, then dimension-reduced feature will be classified using the simple Bias classifier. The tracking algorithm has strong robustness with high accuracy.

\subsection{Compressive Sensing Principle}

The algorithm relates to the theory of compressive sensing (or sparse representation). The basic method is as follows: the signal is sparse (compressibility) in an orthogonal space, the signal is likely to be collected with a lower frequency and can be reconstructed with high probability.

Let $x$ be the one-dimensional signal with a length of $\mathrm{N}$, the sparsity is $\mathrm{K}$ (with $\mathrm{k}$ non-zero value), $\mathrm{A}$ is a $\mathrm{M} \times \mathrm{N}$ matrix $(\mathrm{M}<\mathrm{N}), y=\Phi x$ is measuring value of one dimensional with a length of M. Compressive sensing problem is shown as: when the measure value $y$ and the measurement matrix $\Phi$ is known, to solve underdetermined system of equation $y=\Phi x$ to get original signal $x$. The optimization problem should be solved:

$$
\hat{x}=\arg \min \|x\|_{0} \text { s.t } \Phi x=y
$$

Natural signal $\mathrm{x}$ should be sparse represented on some sparse basement, $x=\Psi s, \Psi$ is sparse basement matrix, $s$ is sparse coefficient. Compressive sensing equation is $y=\Phi x=\Phi \Psi s . \Theta=\Phi \Psi$ (Called the sensing matrix), the approximation solution of $s$ will be calculated, marked as $\hat{s}$, the original signal $=\hat{x} \Psi \hat{s}$.

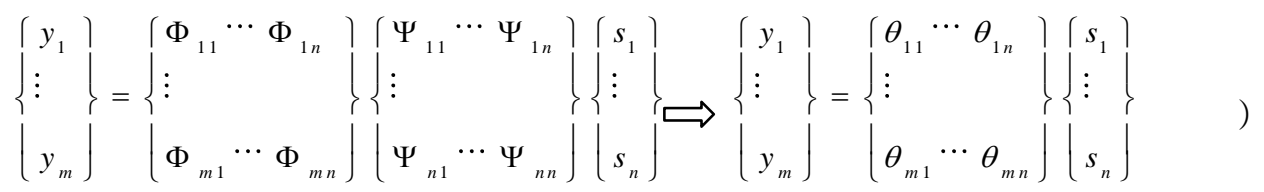

\subsection{Compressive Tracking}

Compressive tracking algorithm requires tracking target to be selected by mouse or read from file, and is not suitable for the traffic light tracking. So we make the adjustment, once 
the classifier identifies the traffic light, the coordinates that has already been identified will be delivery to the tracking algorithm to locate the target. The tracking algorithm is shown below:

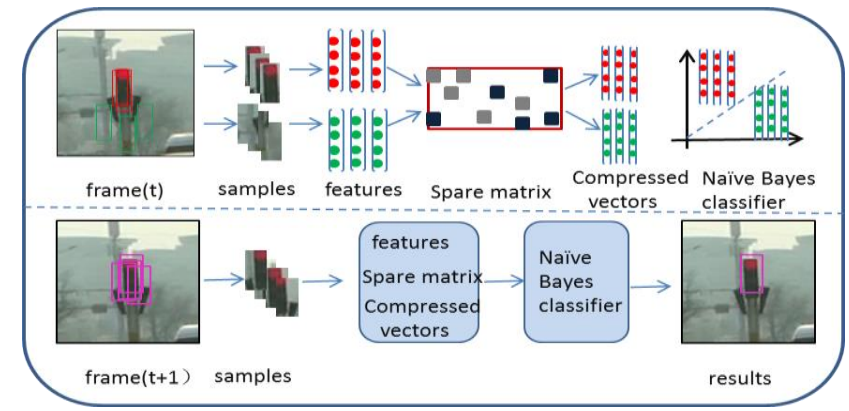

Figure 2.The schematic of the Compressive Tracking

(1)First, obtain the traffic light's position identify by the classifier in the T-1 frame, around the target location $I_{t-1}$ (that is, meet the $D^{\gamma}=\left\{z\left\|\mid 1(\mathrm{z})-1_{t-1}\right\|<\gamma\right\}$, and $1(\mathrm{z})-1$ distance is less than the gamma) sample $\mathrm{n}$ image slices, and then extract feature of the image slice (reduce dimension), get the feature vector $\mathrm{V}$ of each image to train the naive Bayes classifier.

(2)In the $\mathrm{t}$ frame, we extract $\mathrm{n}$ scan windows around the target position we tracked in last frame, then use the same sparse measurement matrices to reduce the dimension, extract the feature, then we use simple Bias classifier which is trained already in $\mathrm{T}-1$ frame to classify, the window with largest classification fraction is considered to be the objective window.

(3) Collect two samples: $D^{\alpha}=\left\{z\left\|1(\mathrm{z})-1_{t-1}\right\|<\alpha\right\}$ and $D^{\varsigma, \beta}=\left\{\varsigma<\left\|1(\mathrm{z})-1_{t}\right\|<\beta\right\}$, $\alpha<\varsigma<\beta$, extract the feature of the two sets of samples, update the classifier parameters.

\subsection{Identification}

The traffic light is different from the common moving target, the target size varies a lot in the process of tracking from far and near, the tracking window size has been determined in the first frame by the tracking compressive algorithm, the tracking window can't change. Aiming at this characteristic, this algorithm is designed as follows: when the same type traffic light is identified within 4 consecutive frames by the classifier, compressive tracking will begin at fifth frame, track 3 frames then identify 1 frame, to ensure the stability and accuracy of recognition.

operation $=\left\{\begin{array}{l}\text { identification, frame } \% 4=1 \& \& \text { fram e }>4 \\ \text { tracking, frame } \% 4 \neq 1 \& \& \text { frame }>4\end{array}\right.$

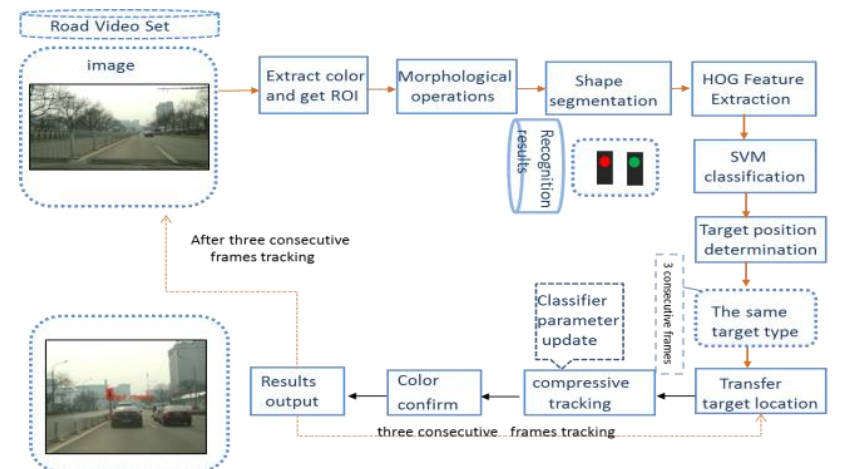

Figure 3. The Schematic of the Overall Algorithm Flow 
Color transformation will appear in the process of tracking, tracking is normal under color conversion situation in this algorithm; there is no phenomenon of tracking drift. In order to avoid color changing which will affect the accuracy in the process of recognition, color recognition of the target will be conducted once the compression tracking is finished. If the color of tracking identifying is the same as what is identified by the classifier, the tracking type of traffic light is the default type of identification. Otherwise, the tracking result information is not sent, and the correct type of traffic light will be conducted by the recognition algorithm of the classifier.

\section{Results and Discussion}

This algorithm has been tested on the real road using the intelligent vehicle in Beijing Union University, and the testing time reaches 2 hours. The development environment is Visual C++2010, and the computer has a CPU with 3.4GHZ and 1GB memory, image resolution is $1000 * 1000$. Each frame processing time of identification part is $250 \mathrm{~ms}$, the tracking part is $40 \mathrm{~ms}, 3-4$ frames will be processed per second in average, and it can meet the requirements of stability and real-time.

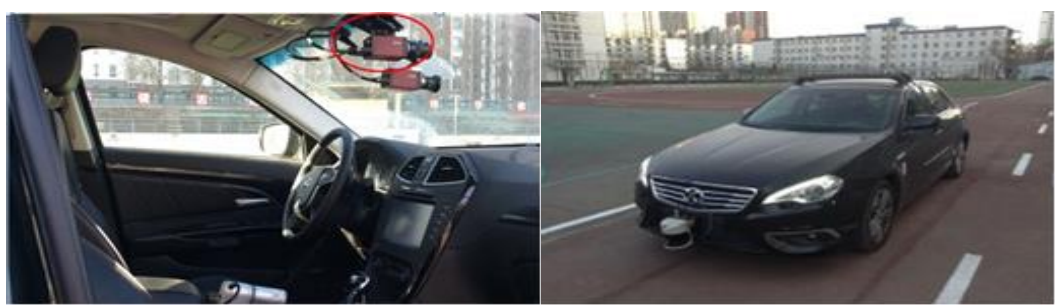

Figure 4. Experimental Environment and Equipment

The results of recognition in this algorithm are shown in figure a, red round represents that the traffic light is red and round. The 3 frames later is the result of continuous tracking. The result of tracking recognition is represented by a blue box, as shown below:

(a)

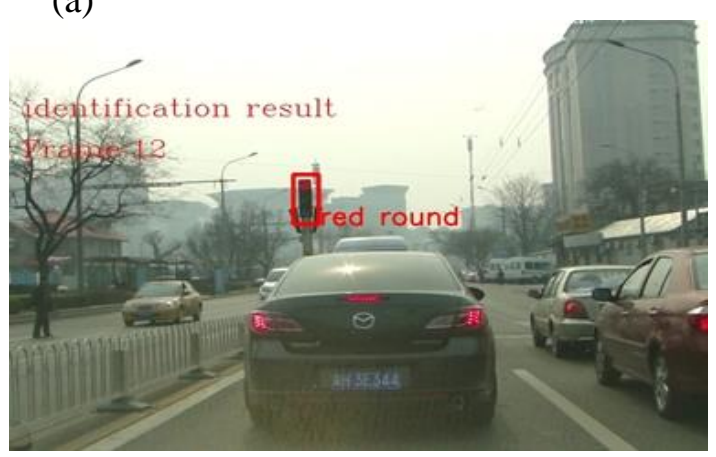

(c)

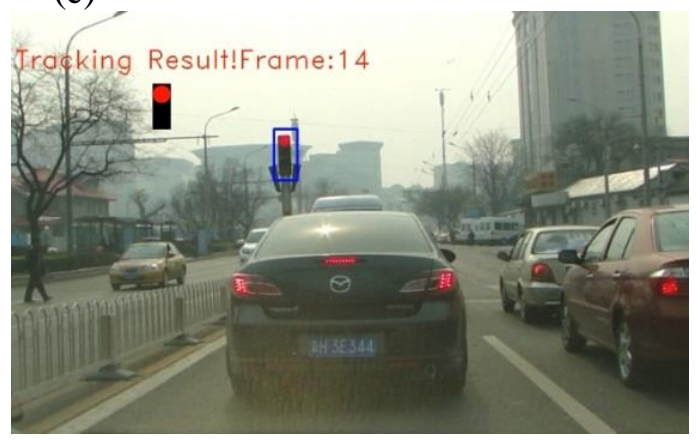

(b)



(d)



Figure 5. The Schematic of Test Results 
The color of the traffic light changes inevitably in the process of tracking. In Figure b, color transformation, target tracking is stable. When the current color is calculated different from red, the tracking information of this frame will not be sent. After the second recognition of diagram c, the accurate identification result will be obtained in diagram $\mathrm{d}$.

(a)

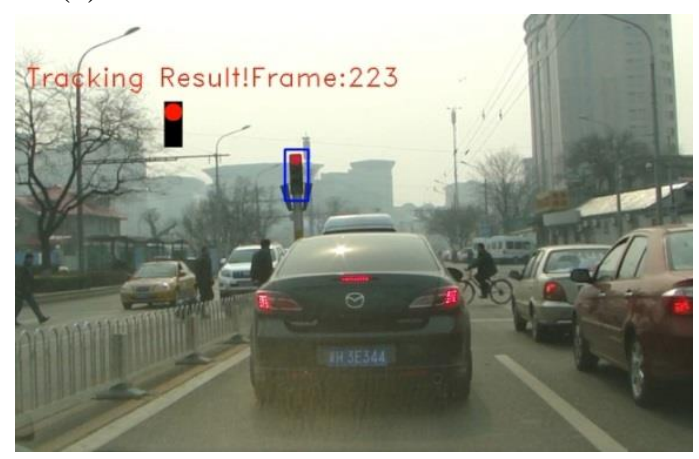

(c)

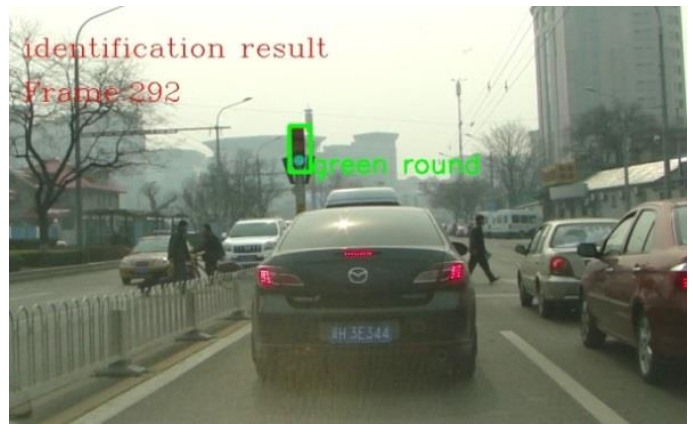

(b)

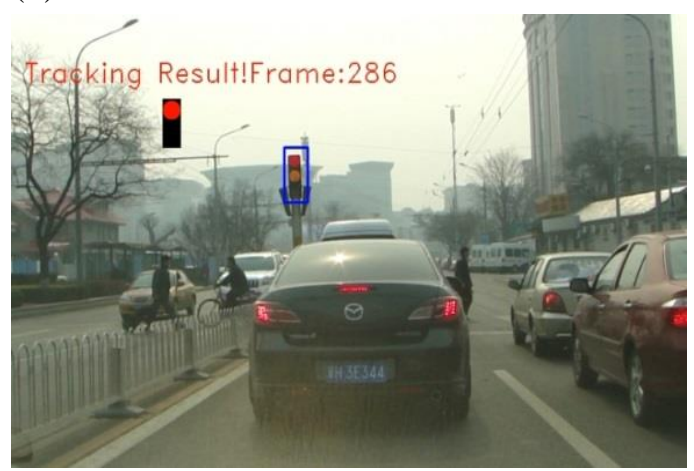

(d)

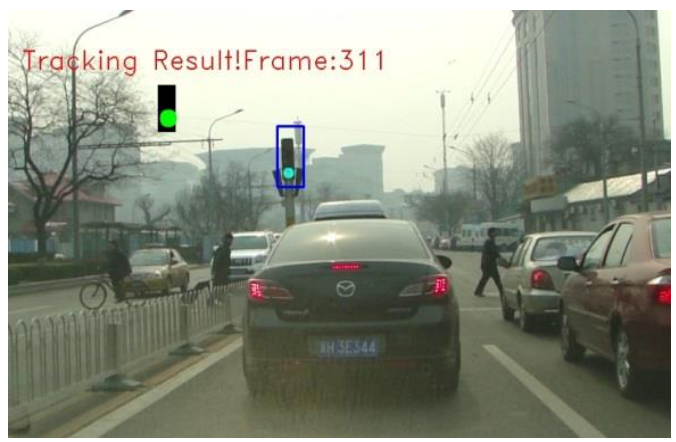

Figure 6. The Schematic of Test Results

The following graph shown below will be a good example when talking about the accuracy of the tracking location in this algorithm. Although the recognition rectangular box shown above can include the traffic light completely, but the size of the box is different. The figure below expresses the position relationship between the coordinate points and the video frame. The coordinate point of this graph is located in the upper left corner of the rectangular box. The result in the figure shows that: the algorithm has a high accuracy, no track loss happens, only the longitudinal coordinate tracking result slightly jumps, but has little effect. The figure is the random result and the specific value has a relation with the speed and distance to the traffic light.

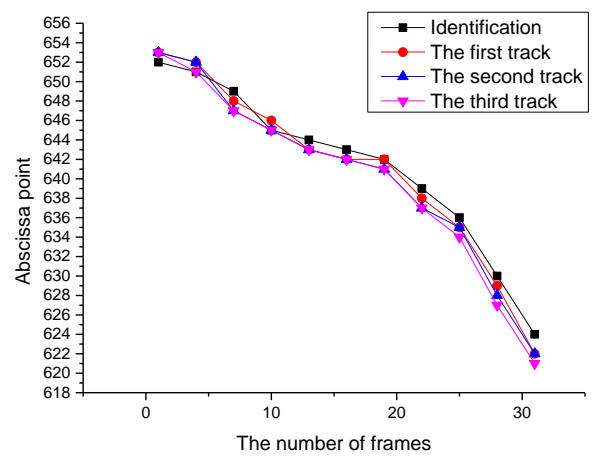

Figure 7. The Schematic of Abscissa Change 


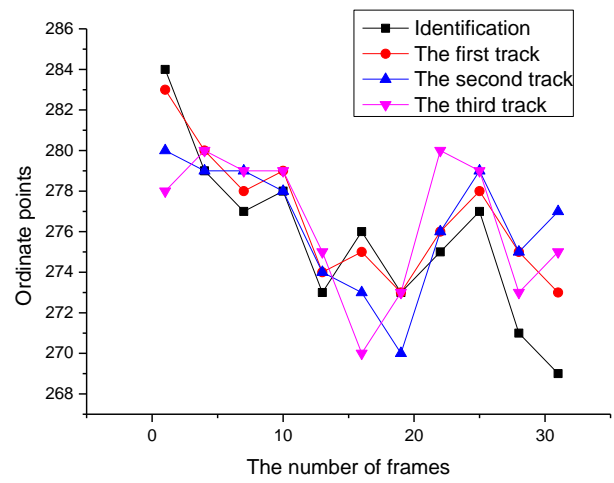

Figure 8. The Schematic of the Vertical Change

\section{Conclusion}

The candidate regions of traffic light is determined using image processing knowledge, and a database is established by collecting a large number of traffic light samples, machine learning method is used to identify. In order to make use of the correlation information between the frame and the frame, compressive tracking is conducted to track and recognize the traffic light. Compared to the simple recognition algorithm, the speed of this algorithm has been improved, the stability is enhanced. According to the characteristics of traffic light and tracking method, method of the feature recognition and tracking recognition alternates, so that identifying windows can adaptively change. The actual road testing for algorithm gets good results. The focus of future research will be how to identify the traffic light accurately at night.

\section{Acknowledgements}

This paper supported by the following projects : (CIT\&TCD20130513); ( IDHT20140508 ) ; ( 2014BAK08B02 ) ; The National Natural Science Foundation of China (No. 61271369), (No. 61372148), (No. 41101111);Funding Project for Academic Human Resources Development in Beijing Union University.

\section{References}

[1] J. Choi, B. T. Ahn and I. S. Kweon, "Crosswalk and traffic light detection via integral framework", 2013 19th Korea-Japan Joint Workshop on Frontiers of Computer Vision, (2013) January 30 - February 01, Incheon, Korea (South).

[2] C. Yu, C. Huang and Y. Lang, "Traffic Light Detection during Day and Night Conditions by a Camera", 2010 IEEE 10th International Conference on Traffic light Processing, (2010) October 24-28, Beijing,China.

[3] M. Diaz-Cabrera, P. Cerri and J. Sanchez-Medina, "Suspended Traffic Lights Detection and Distance Estimation Using Color Features", 2012 15th International IEEE Conference on Intelligent Transportation Systems, (2012) September 16-19, Alaska, USA.

[4] M. R. Yelal, S. Sasi and G. R. Shaffer, "Color-based traffic light tracking in real-time video", Proceedings of the IEEE International Conference on Video and Traffic light Based Surveillance, (2006) November 22-24, Sydney, Australia.

[5] L. Jia, "An Efficient Night Traffic Light Recognition Method", Journal of Information \& Computational Science, vol. 10, no. 9, (2013).

[6] Y. Shen, U. Ozguner and K. Redmill, "A robust video based traffic light detection algorithm for intelligent vehicles”, Intelligent Vehicles Symposium, IEEE, (2009) June 03-05, Xi'an, China.

[7] Y. Jie, C. Xiaomin and G. Pengfei, "A new traffic light detection and recognition algorithm for electronic travel aid", 2013 Fourth International Conference on Intelligent Control and Information Processing (ICICIP), ( 2013 ) June 09-11, Beijing, China.

[8] J. H. Park and C. Jeong, "Real-time traffic light detection", 2008 Second International Conference on Future Generation Communication and Networking Symposia, IEEE, (2008) December 13-15, Hainan Island.

[9] F. Lindner, U. Kressel and S. Kaelberer, "Robust recognition of traffic lights", 2004 IEEE International 
Vehicle Symposium, (2004) June 14-17, Parma, Italy.

[10] M. Omachi and S. Omachi, "Traffic Light Detection with Color and Edge Information", 2009 2nd IEEE International Conference on Computer Science and Information Technology, (2009) August 8-11, Beijing, China.

[11] R. D, Charette and F. Nashashibi, "Real time visual traffic lights recognition based on spot light detection and adaptive traffic lights templates", Intelligent Vehicles Symposium, IEEE, (2009) June 03-05, Xi'an, China.

[12] G. Siogkas, E. Skodras and E. Dermatas, "Traffic Lights Detection in Adverse Conditions using Color, Symmetry and Spatiotemporal Information”, VISAPP-International Conference on Computer Vision Theory and Applications, (2012) February 24-26, Rome, Italy.

[13] C. Wang and T. Jin, "Robust and Real-Time Traffic Lights Recognition in Complex Urban Environments", International Journal of Computational Intelligence Systems, vol. 4, no. 6, (2011).

[14] H. K. Kim, Y. N. Shin and S. Kuk, "Night-Time Traffic Light Detection Based On SVM with Geometric Moment Features", World Academy of Science, Enigineering and Technology, vol. 7, (2013), pp. 6.

[15] C. C. Chiang, M. C. Ho and H. S. Liao "Detecting and recognizing traffic lights by genetic approximate ellipse detection and spatial texture layouts. International Journal of Innovative Computing", Information and Control, vol. 7, (2011), pp. 12.

[16] N. Fairfield and C. Urmson, "Traffic light mapping and detection", 2011 IEEE International Conference on Robotics and Automation (ICRA), (2011) May 9-13, Shanghai, China.

[17] J. Levinson, J. Askeland, J. Dolson, "Traffic light mapping, localization, and state detection for autonomous vehicles. 2011 IEEE International Conference on Robotics and Automation (ICRA), (2011) May 9-13, Shanghai, China.

[18] G. Li and Z. Huang, "Particle filter based railway traffic lights tracking", Journal of Sichuan University (Natural Science Edition), vol. 50 , (2013), pp. 2

[19] J. Gong, Y. Jiang and G. Xiong, "The recognition and tracking of traffic lights based on color segmentation and camshift for intelligent vehicles", Intelligent Vehicles Symposium (IV), (2010) June 21-24, California, San Diego.

[20] K. Zhang, L. Zhang and M. H. Yang, "Real-time compressive tracking", Computer Vision-ECCV 2012, (2012) October 7-13, Florence, Italy.

Authors

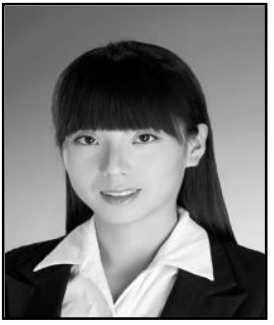

Xuanru Zhou, She is now a master of Beijing Union University and her main research directions is Graphic image processing



Jiazheng Yuan, He recived his Ph.D. in computer science from Beijing Jiaotong University in 2007. He is now a professor in Beijing Key Laboratory of Information Service Engineering, Beijing Union University, and Beijing, China. His current research interests include graph and image processing, digital processing of cultural relics, digital museum.

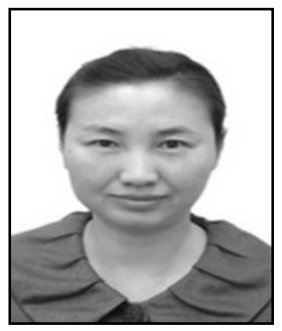

Hongzhe Liu, She recived her Ph.D. in computer science from Beijing Jiaotong University in 2012. She is now a associate professor in Beijing Key Laboratory of Information Service Engineering, Beijing Union University, Beijing, China. Her current research interests include digital museum, semantic calculating and image processing. 
International Journal of Hybrid Information Technology Vol.8, No.6 (2015) 